\title{
A Quasi-panoramic Bio-inspired Eye for Flying Parallel to Walls
}

\author{
Erik Vanhoutte, Franck Ruffier, and Julien Serres ${ }^{1}$
}

\begin{abstract}
In this study, a quasi-panoramic bio-inspired eye dedicated to optic flow measurement on board micro flying robots is presented. It will allow future micro flying robots to mimic honeybees' navigational tasks which work without any metric sensors. An innovative optic flow-based algorithm was tested in the horizontal plane to measure the robot's incidence angle when travelling along a wall. Experimental results achieved while using a moving texture showed that our algorithm is able to measure the local visual heading with respect to a tapered wall with an error of $3^{\circ}$ and an accuracy of $3^{\circ}$ on average. Our bio-inspired eye will be implemented in the near future on board a micro quadrotor to "sense and avoid" obstacles in a GPS-denied environment.
\end{abstract}

\section{INTRODUCTION}

Detailed study of flying insects has led to the discovery of smart solutions in biologically inspired robotics [13]. One of the most impressive flying insects is the honeybee because it is able to navigate up to a few kilometers from its hive to forage without access to GPS (GPS stands for Global Positioning System) while avoiding obstacles mainly through the use of optic flow (OF) sensing [13]. Since, different mobile robots ([6], [3], [13]) or micro flying robots ([1], [10], [5], [2]), fitted with a bio-inspired custom-made compound eyes to measure OF, have been built for indoor navigation in obstacle-rich corridors or forests.

OF-based control methods have been developed on board simulated ([8], [7], [11]) or physical micro flying robots ([1], [10]) in order to follow flat surfaces (ground or walls). However, OF-based strategy has been also demonstrated to improve the crossing capabilities in case of a tapered wall encountered in bent corridors [12]. In [4], the slope of the ground has been assessed on board a quadrotor. In [5], 10 local OF measurements coupled to a least mean squares method was used to keep constantly level a minimalistic quasi-panoramic compound eye of the Beerotor robot with respect to the local downward slope, thus allowing the robot to avoid very steep relief.

In the present paper, a quasi-panoramic bio-inspired eye built with 8 custom-made OF sensors (based on $\mathrm{M}^{2} \mathrm{APix}$ sensor [9], [14]) is presented for local visual heading measurement purposes : it should allow a future micro flying robot devoid of a magnetic compass to stabilize its heading in an unpredictable indoor environment while visually following local flat surfaces. In section II, the eye design will be

*This work was supported by the French Direction Générale de l'Armement (DGA), CNRS, Aix-Marseille Université, the Provence-AlpesCôte d'Azur region and the French National Research Agency for Research (ANR) with the Equipex/Robotex project.

${ }^{1}$ Correspondance: julien.serres@univ-amu.fr; Tel.: +33(0)4 91266238 Aix Marseille Univ, CNRS, ISM, Marseille, France described. In section III, both the visual signals processing and the local visual heading computation on OF sensing will be presented. In section IV, the embedded electronics for computational purposes will be described in detail, and in section $\mathrm{V}$, experimental results obtained while using a test bench based on a moving texture will be presented to test our visual heading estimation algorithm. In section VI, it will be concluded that our quasi-panoramic bio-inspired eye is able to estimate the local visual heading profile with respect to a tapered wall set in motion.

\section{QUASI-PANORAMIC BIO-INSPIRED EYE}

A custom-made 3D print gimbal case was designed to be used on board our micro flying robot to support our bioinspired eye (Fig. 1). Two 10-gram servo motors mounted in serial stabilize the 8 OF sensors (based on $\mathrm{M}^{2} \mathrm{APix}$ sensor [9], [14]) in pitch and roll (Fig. 1) to avoid any perturbation coming from attitude disturbances. A first group of $3 \mathrm{OF}$ sensors are oriented toward the left-hand side, a second group of 3 sensors is oriented toward the right-hand side. Each group of sensors placed in the horizontal plane has a field of view of $92.8^{\circ}$ in the horizontal plane and $13.4^{\circ}$ in the vertical plane.

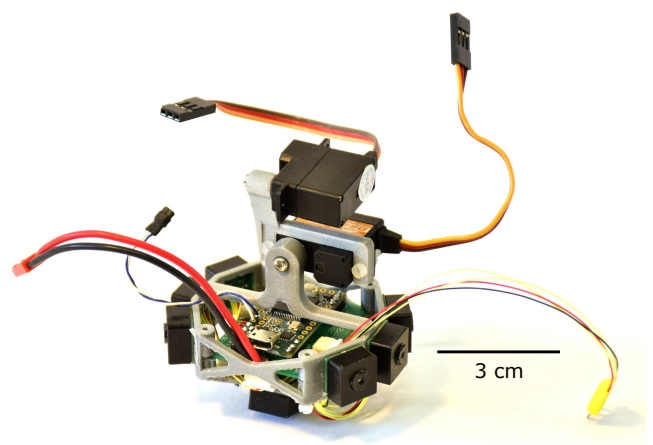

Fig. 1: The 50-gram bio-inspired eye called OctoM ${ }^{2}$ APix.

A third group of 2 sensors is oriented downwards to measure the ventral OF with a field of view of $62.8^{\circ}$ in the forward axis and $13.4^{\circ}$ in the orthogonal axis. This quasi-panoramic bio-inspired eye was designed to provide navigational capabilities to a micro flying robot (see [14]) in order to adopt wall-following behaviour in indoor environments while following the ground by regulating the OF. Moreover, 10-local OF measurements is obtained by each $\mathrm{M}^{2} \mathrm{APix}$ sensor, and each of these pixels is based on a Michaelis-Menten Auto-adaptive Pixel analog silicon retina that can auto-adapt in a 7-decade lighting range and responds appropriately to step changes up to \pm 3 decades 


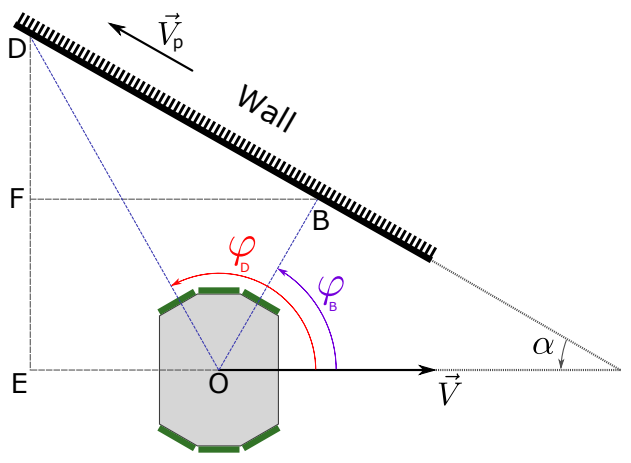

Fig. 2: Geometry of the Octo $\mathrm{M}^{2} \mathrm{APix}$ eye (centering in $\mathrm{O}$ ) in the horizontal plane and a tapered wall on its left-hand side. Each green rectangle represents one $\mathrm{M}^{2} \mathrm{APix}$ sensor.

[9]. Consequently, a $\mathrm{M}^{2} \mathrm{APix}$ sensor can work in any lighting conditions [14].

\section{VISUAL HEADING COMPUTED WITH OPTIC FLOW}

In our experiment the bio-inspired eye, called OctoM $^{2}$ APix (Fig. 1), is placed in front of a moving pattern to perceive an $\mathrm{OF}$ with its 3 left-hand $\mathrm{M}^{2} \mathrm{APix}$ sensors (same experimental set-up as Fig. 4 in [14]). Different possibilities are available to compute the local visual heading $\alpha$ from a tilted surface (here, a wall), but one of the lightest solution in terms of CPU load is to consider a median filter from the 10 local OF measurements coming from each $\mathrm{M}^{2} \mathrm{APix}$ sensor. Hence, the local visual heading $\alpha$ can be computed with only 2 local OF measurements $\left(\omega_{B}\right.$ and $\left.\omega_{D}\right)$ and their respective azimuthal angle $\left(\varphi_{B}\right.$ and $\varphi_{D}$ in Fig. 2). The computed angle $\alpha$ will be independent from the robot's forward speed $V$ (Eq. (1)). However, the bio-inspired eye will be immobile in space while seeing a moving texture at a speed $V_{p}$, thus requiring a correction Eq. (1) by mean of Eq. (2). With a speed condition $V_{p}=0$ and $V \neq 0$ (real flight), the local visual heading $\alpha$ is computed with Eq. (1), where $c$ is $\cos , s$ is sin, and $t$ is tan function. However, with a speed condition $V_{p} \neq 0$ and $V=0$, the local visual heading $\alpha$ is computed with Eq. (2).

$$
\begin{aligned}
\omega_{B} & =\frac{V}{O B} \cdot s\left(\varphi_{B}\right) \quad \omega_{D}=\frac{V}{O D} \cdot s\left(\varphi_{D}\right) \\
t(\alpha) & =\frac{O D \cdot s\left(\varphi_{D}\right)-O B \cdot s\left(\varphi_{B}\right)}{O B \cdot c\left(\varphi_{D}\right)-O D \cdot c\left(\varphi_{B}\right)} \\
t(\alpha) & =\frac{\omega_{B} \cdot s\left(\varphi_{D}\right)^{2}-\omega_{D} \cdot s\left(\varphi_{B}\right)^{2}}{\omega_{D} \cdot s\left(\varphi_{B}\right) \cdot c\left(\varphi_{B}\right)-\omega_{B} \cdot s\left(\varphi_{D}\right) \cdot c\left(\varphi_{D}\right)} \\
t(\alpha) & =\frac{\omega_{B} \cdot s\left(\varphi_{D}+\alpha\right) \cdot s\left(\varphi_{D}\right)-\omega_{D} \cdot s\left(\varphi_{B}+\alpha\right) \cdot s\left(\varphi_{B}\right)}{\omega_{D} \cdot s\left(\varphi_{B}+\alpha\right) \cdot c\left(\varphi_{B}\right)-\omega_{B} \cdot s\left(\varphi_{D}+\alpha\right) \cdot c\left(\varphi_{D}\right)}
\end{aligned}
$$

Our algorithm uses a pair of local OF measurements (Eq. (2)) among $3 \mathrm{M}^{2}$ APix sensors, 3 local visual heading $\alpha$ can be therefore computed and compared.

\section{EMBEDdEd ELECTRONICS}

A custom-made PCB was designed to connect, as presented in the Fig. 3a, the following components: a Teensy 3.2 featuring a $72 \mathrm{MHz}$ Cortex-M4 (PJRC), an 8-bit SPI GPIO EXPANDER called XRA1404 (Exar@), an Inertial
Measurement Unit (IMU) MPU6050, and connections to plug $8 \mathrm{M}^{2}$ APix sensors and 2 servo motors of the gimbal system which aims to stabilize the bio-inspired eye in pitch and roll.

A $\mathrm{M}^{2}$ APix sensor works with a SPI bus running at maximum $1 \mathrm{MHz}$ to transmit a data frame of 256 bits. The maximum theoretical frame rate is at $3906 \mathrm{~Hz}$. SPI devices communicate in full duplex mode using a masterslave architecture with a single master. By and large, only one SPI bus is available on dedicated electronic board. So, the maximum frame rate to get data from 8 slave $\mathrm{M}^{2} \mathrm{APix}$ sensors is less than $488 \mathrm{~Hz}$, and data will not process synchronously but sequentially (Fig. 3b). To get a higher frame rate from $8 \mathrm{M}^{2} \mathrm{APix}$ sensors, and to be synchronous, a XRA1404 GPIO EXPANDER was used. The XRA1404 can read the logic state on 8 digital ports and send this data as a byte to a SPI bus working at $26 \mathrm{MHz}$ (Fig. 3b). Each of these 8 digital ports is used to read the bit to bit data frame of each $\mathrm{M}^{2}$ APix sensor (step 1 in the Fig. $3 b$ ). As a result, the first bit of each $\mathrm{M}^{2} \mathrm{APix}$ data frame is grouped in one byte that is sent on the SPI bus. Each bit of each $\mathrm{M}^{2} \mathrm{APix}$ sensor is processed in this way.

The Teensy electronic board reads the SPI bus at 26 MHz from the XRA1404 and puts each data frame of each $\mathrm{M}^{2} \mathrm{APix}$ sensor back in order (step 2 in Fig. 3b). The $\mathrm{M}^{2} \mathrm{APix}$ sensor sends a mean value of light and 12 pixel values, each one coded on 10-bit values. Then, the algorithm selects only the pixel data. It remains $12 \times 10$ bits per $\mathrm{M}^{2} \mathrm{APix}$ sensor . Finally, the Teensy removes the offset of the used range and deletes the last bit equivalent to the amplitude of the noise to get data in the 8-bit format. Then, the 12 pixels coded with the 8-bit value of the $8 \mathrm{M}^{2} \mathrm{APix}$ sensors are sent to the serial bus at 3 Mbps to a computational unit to compute OF (step 3 in Fig. 3b).

\section{RESUlTS WITH A MOVING TEXTURE AND CONCLUSION}

Results of the local visual heading $\alpha$ computed with Eq. (2) with the 3 pairs of left-hand $\mathrm{M}^{2} \mathrm{APix}$ sensors in four $\alpha$ angle cases are presented in Fig. 4. The eye heading is manually rotated along the yaw axis with an accuracy of $\pm 3^{\circ}$, and it is represented by 4 purple steps in Fig. 4 . Using Eq. (1) assumes the hypothesis of a flying robot at a speed $\vec{V}$ (Fig. 2), but in this experiment, the wall was moving at a speed $\overrightarrow{V p}$ (Fig. 2), which is not collinear with $\vec{V}$, so a correction has been made offline to compute the correct visual heading $\alpha$ with Eq. (2).

These results show that such a group of OF sensors (here 20 local OF measurements) can be used on board a flying robot to implement a yaw control feedback loop based on a local visual heading estimation (error less than $3^{\circ}$ in Fig. 4). Such bio-inspired eyes will be used in the future when exploring a building by keeping a visual contact with the wall while keeping the quadrotor heading parallel to the same wall [15]. Moreover, the computation of OF and local visual heading appears to be light enough in terms of computational resources to be embedded on board a micro flying robot fitted 
a)

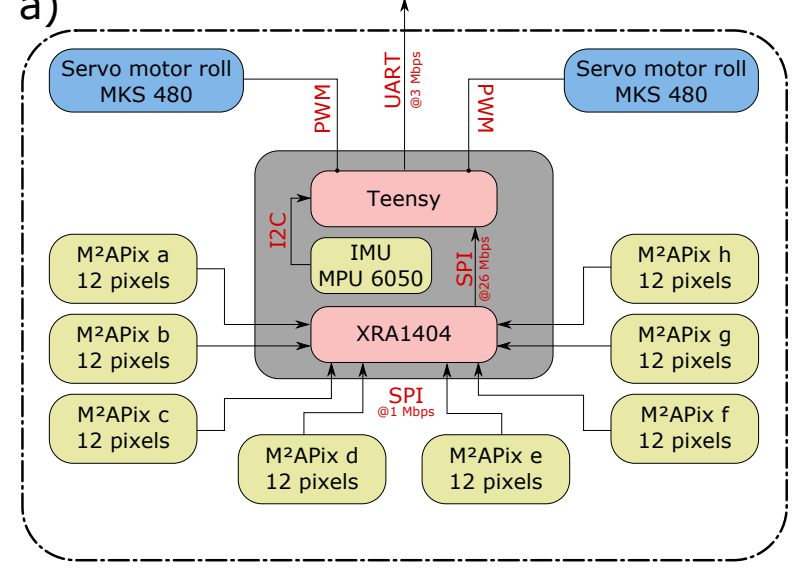

b)

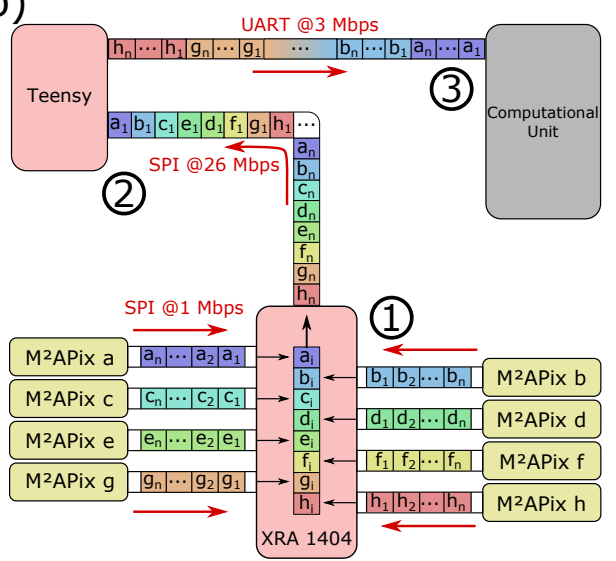

Fig. 3: a) Electronic diagram. b) Data diagram of how visual signals coming from the OctoM ${ }^{2}$ APix eye are processed.

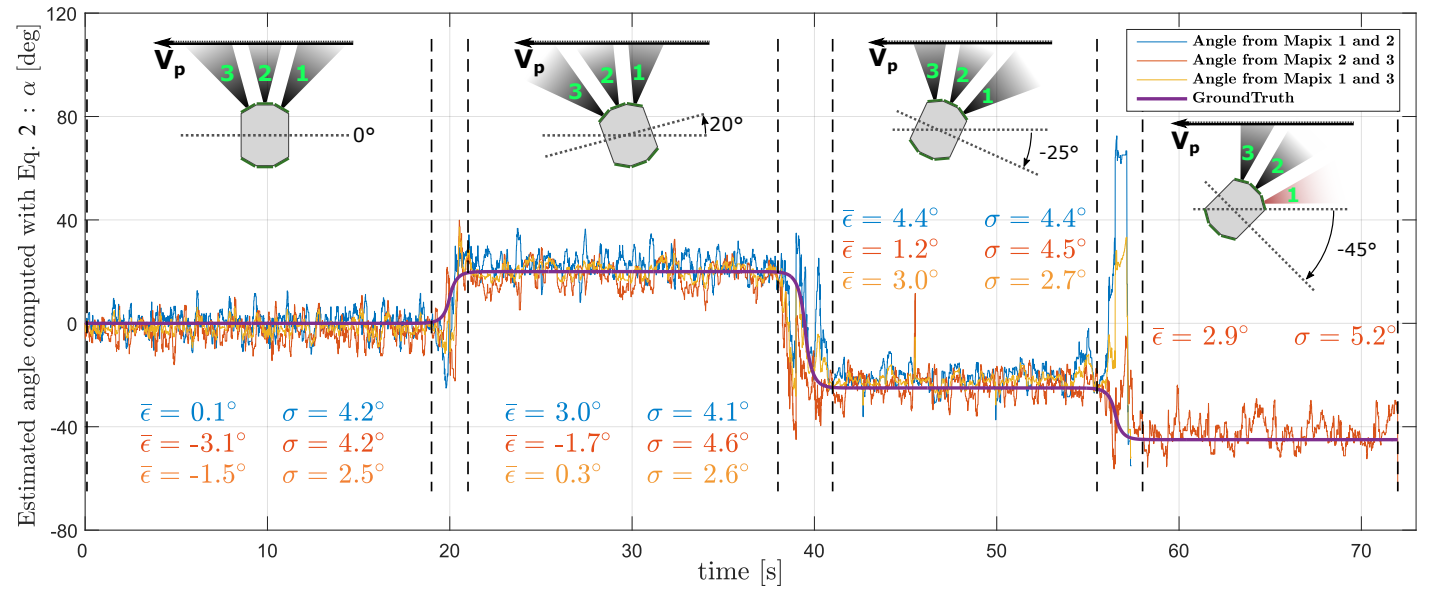

Fig. 4: Incidence angle assessment with respect to a textured wall set in motion using the OctoM ${ }^{2} \mathrm{APix}$ eyes. Results are shown for 4 different incidence angles $\alpha: 20 \mathrm{deg},-25 \mathrm{deg}$ and $-45 \mathrm{deg}$. The incidence angle estimation was compared in the case of three different combinations of $\mathrm{M}^{2} \mathrm{APix}$ sensors: numbered from 1 to 3. Precision and accuracy are in general the lowest while using the pair of sensors 1 and 3 , the most widely separated ones.

with an Overo AirSTORM Computer-On-Module (COM) (Gumstix) including a 1-GHz CPU DM3703 processor.

\section{REFERENCES}

[1] Autonomous Vision-Based Navigation of a Quadrotor in Corridor-Like Environments. International Journal of Micro Air Vehicules, 7(2):111124, 2015.

[2] A. Briod, J.-C. Zufferey, and D. Floreano. A method for egomotion estimation in micro-hovering platforms flying in very cluttered environments. Autonomous Robots, 40(5):789-803, 2016.

[3] J. Davis, S. Barrett, C. Wright, and M. Wilcox. A bio-inspired apposition compound eye machine vision sensor system. Bioinspiration \& biomimetics, 4(4):046002, 2009.

[4] G. De Croon, H. Ho, C. De Wagter, E. Van Kampen, B. Remes, and Q. Chu. Optic-flow based slope estimation for autonomous landing. International Journal of Micro Air Vehicles, 5(4):287-297, 2013.

[5] F. Expert and F. Ruffier. Flying over uneven moving terrain based on optic-flow cues without any need for reference frames or accelerometers. Bioinspiration and Biomimetics, 10, 2015.

[6] N. Franceschini, J.-M. Pichon, C. Blanes, and J. Brady. From insect vision to robot vision [and discussion]. Philosophical Transactions of the Royal Society of London B: Biological Sciences, 337(1281):283294, 1992.

[7] J. Humbert and M. Frye. Extracting behaviorally relevant retinal image motion cues via wide-field integration. 2006 American Control Conference, 1(3):2724-2729, 2006.
[8] J. S. Humbert, R. M. Murray, and M. H. Dickinson. A control-oriented analysis of bio-inspired visuomotor convergence. In Decision and Control, 2005 and 2005 European Control Conference. CDC-ECC'05. 44th IEEE Conference on, pages 245-250. IEEE, 2005.

[9] S. Mafrica, S. Godiot, M. Menouni, M. Boyron, F. Expert, R. Juston, N. Marchand, F. Ruffier, and S. Viollet. A bio-inspired analog silicon retina with Michaelis-Menten auto-adaptive pixels sensitive to small and large changes in light. Optics Express, 23(5):5614, 2015.

[10] R. J. Moore, K. Dantu, G. L. Barrows, and R. Nagpal. Autonomous mav guidance with a lightweight omnidirectional vision sensor. In 2014 IEEE International Conference on Robotics and Automation (ICRA), pages 3856-3861. IEEE, 2014.

[11] G. Portelli, J. Serres, F. Ruffier, and N. Franceschini. Modelling honeybee visual guidance in a 3-D environment. Journal of Physiology Paris, 104(1-2):27-39, 2010.

[12] J. R. Serres and F. Ruffier. Biomimetic Autopilot Based on Minimalistic Motion Vision for Navigating along Corridors Comprising Ushaped and S-shaped Turns. Journal of Bionic Engineering, 12(1):4760, jan 2015 .

[13] M. V. Srinivasan. Honeybees as a model for the study of visually guided flight, navigation, and biologically inspired robotics. Physiological reviews, 91(2):413-460, 2011.

[14] E. Vanhoutte, S. Mafrica, F. Ruffier, R. Bootsma, and J. Serres. Timeof-Travel Methods for Measuring Optical Flow on Board a Micro Flying Robot. Sensors, 17(3):571, 2017.

[15] E. Vanhoutte, F. Ruffier, and J. Serres. A honeybee's navigational toolkit on board a bio-inspired micro flying robot. In IMAV 2017, International Micro Air Vehicle Conference and Competition 2017, paper 14, Toulouse, France, 2017 (in press). 